\title{
Carcinosarcoma with a large cell neuroendocrine epithelial component: first report of an unusual biphasic tumour of the urinary bladder
}

\author{
Y Li, J E Outman, S C Mathur
}

J Clin Pathol 2004;57:318-320. doi: 10.1136/icp.2003.013474

This article describes the first reported case of carcinosarcoma of the urinary bladder with a large cell neuroendocrine epithelial component. A 61 year old man presented with gross haematuria and underwent resection of a biphasic bladder tumour. The malignant epithelial component showed large cell neuroendocrine differentiation with immunohistochemical reactivity for neurone specific enolase, synaptophysin, and chromogranin. The malignant mesenchymal component did not show specific differentiation by histological or immunohistochemical examination. The differential diagnosis of biphasic tumours in the urinary bladder is discussed, along with a review of the literature.

M alignant tumours in the urinary bladder with a biphasic pattern are rare, and include sarcomatoid carcinoma, carcinoma with pseudosarcomatous stroma, and carcinosarcoma. The epithelial components of these tumours are usually composed of transitional cell carcinoma, squamous cell carcinoma, adenocarcinoma, or undifferentiated carcinoma. Here, we report a case of carcinosarcoma with large cell neuroendocrine epithelial differentiation.

\section{CASE REPORT}

A 61 year old man presented with a two and a half month history of gross haematuria and irritative voiding symptoms. He was initially treated with a course of antibiotics and evaluated with an intravenous pyelogram, which revealed no upper or lower urinary tract pathology. Completion of his evaluation with cystourethroscopy revealed a mass on the right lateral bladder wall. The patient had no previous history of bladder or other organ neoplasia. He had a history of chronic obstructive pulmonary disease and cigarette use. He had no history of occupational exposure to known carcinogens. The patient underwent transurethral resection of the bladder tumour. Based on the pathological diagnosis of carcinosarcoma, he subsequently had a radical cystoprostatectomy with ileal conduit. There was no evidence of metastatic disease and he did not receive additional treatment. The patient was alive with no evidence of disease eight months later.

\section{PATHOLOGY}

Formalin fixed, paraffin wax embedded tissue blocks were sectioned at $4 \mu \mathrm{m}$ and stained with haematoxylin and eosin. Immunohistochemical stains were performed using commercially available primary antibodies (table 1) against epithelial, neuroendocrine, and mesenchymal antigens. Secondary antibody conjugated with peroxidase labelled polymer (EnVision Plus System; Dako, Carpinteria, California, USA) was applied, with 3,3'-diameinobenzidine as chromogen, and the sections were counterstained with haematoxylin. Prior antigen retrieval was performed for some antibodies using a citric acid based solution (Citra System; BioGenex, San Ramon, California, USA) and/or microwave heat. Appropriate positive and negative controls were evaluated and were satisfactory.

Transurethral resection of the bladder tumour yielded $40 \mathrm{~g}$ of intact and necrotic tan to light pink soft tissue fragments. Haematoxylin and eosin stained sections showed a tumour consisting of both malignant epithelial and mesenchymal components (fig 1A,B). The epithelial component consisted of nests and sheets of malignant cells with a high nuclear to cytoplasm ratio, coarse granular chromatin, and scant cytoplasm. Nucleoli were inconspicuous or absent. These cells were distinct from the mesenchymal component that consisted of variably loose and dense sheets of spindle cells with pleomorphic, hyperchromatic nuclei, and occasional prominent nucleoli. Scattered markedly pleomorphic and giant cells were also noted. Mitotic activity was brisk in both components, with occasional atypical forms. Large areas of necrosis were present. The tumour invaded the mural muscle (muscularis propria). Immunohistochemical stains (table 1) showed that the epithelial component was positive for cytokeratin (AE1/AE3 and CAM5.2) and neuroendocrine markers (neurone specific enolase, chromogranin, and synaptophysin) (fig lC). The mesenchymal component was strongly positive for vimentin (fig ID) and focally weakly positive for smooth muscle actin and S-100 protein. Epithelial and neuroendocrine markers were negative in the mesenchymal component. Stains for desmin, muscle specific actin, CD34, and prostate specific antigen were also negative in malignant epithelial and mesenchymal cells. The subsequent radical cystoprostatectomy specimen showed a $2.0 \times 0.6 \times 0.3 \mathrm{~cm}$ defect in the right lateral wall. No residual tumour was identified.

\section{DISCUSSION}

Carcinosarcoma is a rare tumour of the urinary bladder, with fewer than 100 documented cases in the literature. ${ }^{1}$ It occurs more frequently in men (male to female ratio of 1.7-3.0 to 1 ) and is generally seen in older adults, with a mean age in the 7th decade. ${ }^{12}$ The tumours are usually large and polypoid, and on microscopic examination contain an intimate admixture of malignant epithelial and mesenchymal elements. The epithelial components are usually transitional cell carcinoma, squamous cell carcinoma, adenocarcinoma, or undifferentiated carcinoma. The mesenchymal components may be fibrosarcoma, leiomyosarcoma, or undifferentiated sarcoma; rarely skeletal muscle, osseous, and/or cartilaginous differentiation can be seen.

Theories on the histogenesis of carcinosarcoma focus either on divergent differentiation or on "collision" tumours. 
Table 1 Results of immunohistochemical stains

\begin{tabular}{|c|c|c|c|c|}
\hline Stain (clone) & Source & Dilution & Epithelial component & $\begin{array}{l}\text { Mesenchymal } \\
\text { component }\end{array}$ \\
\hline CK (AE1/AE3) & CellMarque (Hot Springs, Arkansas, USA) & $1 / 150$ & +++ & - \\
\hline CK (Cam 5.2) & $\begin{array}{l}\text { Zymed (South San Francisco, California, } \\
\text { USA) }\end{array}$ & $1 / 100$ & +++ & - \\
\hline NSE (BBS/NC/VI-H14) & CellMarque & $1 / 50$ & +++ & - \\
\hline Synaptophysin (SY38) & Biogenex & Prediluted & +++ & - \\
\hline Chromogranin (LK2H10) & CellMarque & $1 / 100$ & +++ & - \\
\hline PSA (ER-PR8) & CellMarque & $1 / 100$ & - & - \\
\hline Vimentin (V9) & CellMarque & $1 / 80$ & - & +++ \\
\hline SMA (1A4) & CellMarque & $1 / 100$ & - & $\pm(w f)$ \\
\hline MSA (HHF35) & DAKO & Prediluted & - & - \\
\hline Desmin (D33) & CellMarque & Prediluted & - & - \\
\hline S-100 (4C4.9) & CellMarque & $1 / 200$ & - & \pm (wf) \\
\hline CD34 (QBEnd/10) & BioGenex & $1 / 30$ & - & - \\
\hline
\end{tabular}

+++ strong, diffuse positive; \pm (wf) weak, focal positive; - negative.

CK, cytokeratin; MSA, muscle specific actin; NSE, neurone specific enolase; PSA, prostate specific antigen; SMA, smooth muscle actin.

Results of immunohistochemistry have been inconclusive, because epithelial markers may or may not be expressed in the sarcomatoid component. Recent studies investigating loss of heterozygosity ${ }^{3}$ show overlapping shared losses between the carcinomatous and sarcomatous elements. This favours an origin from a common multipotential cell, with divergent differentiation. Similar findings have been reported in carcinosarcoma of other sites.

The differential diagnosis of carcinosarcoma includes spindle cell carcinoma (sarcomatoid carcinoma) and carcinoma with pseudosarcomatous stromal reaction. ${ }^{5}$ The presence of urothelial carcinoma in situ and zones of transition between epithelial and mesenchymal cells supports the diagnosis of sarcomatoid carcinoma. In addition, immunohistochemical stains show positivity for epithelial markers in the spindle cells of sarcomatoid carcinoma. Carcinosarcoma can be distinguished from carcinoma with pseudosarcomatous stromal reaction by strict application of criteria for malignancy in the stromal component. An inflammatory background is more likely in pseudosarcomatous stroma, whereas brisk mitotic activity or the presence of atypical mitoses favours a diagnosis of carcinosarcoma.
"Our case is unique in that the epithelial component exhibited large cell neuroendocrine morphology and, to our knowledge, this represents the first report of a carcinosarcoma of the urinary bladder with a large cell neuroendocrine epithelial component"

Carcinosarcoma with neuroendocrine differentiation of the epithelial component is extremely rare, with only a few reports in the literature ${ }^{67}$ Mills and colleagues ${ }^{7}$ studied 12 cases of small cell carcinoma of the urinary bladder and found one case with a sarcomatoid spindle cell component. However, this report did not include detailed immunohistochemical staining results on the spindle cell component. Mazzucchelli and colleagues ${ }^{6}$ reported two cases that had a small cell neuroendocrine epithelial component, confirmed by immunohistochemical stains, and a mesenchymal component with smooth muscle differentiation. Both cases also had foci of transitional cell carcinoma in situ. In contrast to their cases, we did not find carcinoma in situ in either the transurethral resection specimen or cystoprostatectomy specimen from our case. An ultrastructural study was performed in one case in Mazzucchelli's report, which
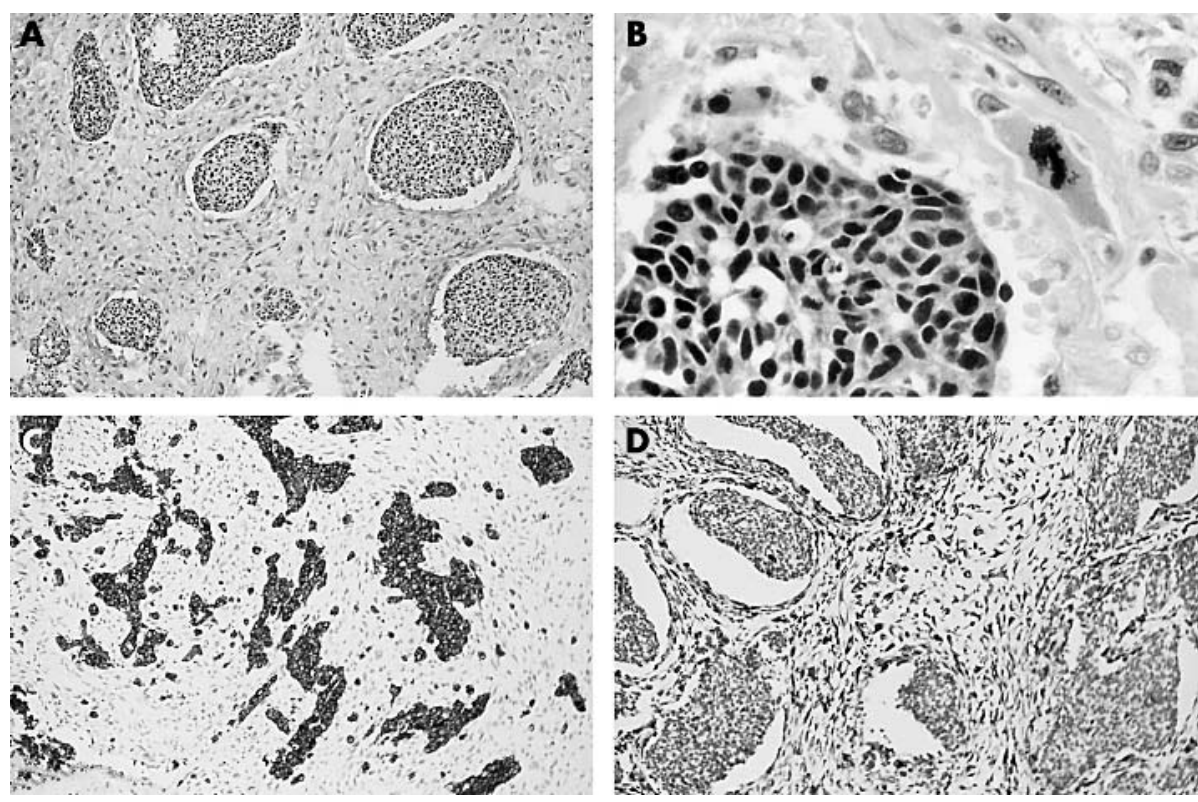

Figure 1 (A) Nests of malignant epithelial cells sharply demarcated from malignant mesenchymal cells; original magnification, $\times 25$. (B) Coarse chromatin without obvious nucleoli in the malignant epithelial cells and an atypical mitotic figure in the mesenchymal component; original magnification, $\times 180$. (C) Immunohistochemical staining for synaptophysin showing positive staining in the epithelial component only; original magnification, $\times 25$. (D) Immunohistochemical staining for vimentin showing positive staining in the mesenchymal component only; original magnification, $\times 25$. 


\section{Take home messages}

- We present the first reported case of carcinosarcoma of the urinary bladder with a large cell neuroendocrine epithelial component

- This rare tumour should be included in the differential diagnosis of biphasic neoplasms of the urinary bladder

- Although carcinosarcomas usually have an aggressive course, the prognosis of carcinosarcoma with epithelial neuroendocrine differentiation in the urinary bladder is unknown, and our patient is alive with no evidence of disease eight months after diagnosis

showed epithelial cells with irregular nuclei, clumped chromatin, and membrane limited neurosecretory granules. Ultrastructural evaluation from paraffin wax embedded tissue was unsuccessful in our case.

Our case is unique in that the epithelial component exhibited large cell neuroendocrine morphology and, to our knowledge, this represents the first report of a carcinosarcoma of the urinary bladder with a large cell neuroendocrine epithelial component. Primary large cell neuroendocrine carcinoma is extremely rare in the bladder, and has been reviewed in a recent report. ${ }^{8}$ Criteria for the diagnosis of large cell neuroendocrine carcinoma were proposed by Travis and colleagues $^{9}$ for tumours of the lung. The epithelial component of our case met these criteria, with areas of nested and trabecular (neuroendocrine) growth, large cell size, coarse nuclear chromatin, high mitotic rate $(>10$ for each 10 high power fields), large areas of necrosis, and immunohistochemical documentation of neuroendocrine differentiation (positivity for synaptophysin, chromogranin, and neurone specific enolase).

Carcinosarcomas are characteristically deeply infiltrative at initial presentation and generally have an aggressive course. In one series, $50 \%$ of patients died of their disease. ${ }^{2}$ Patients usually die from complications of local growth rather than metastases. ${ }^{10}$ The clinical course and prognosis of carcinosarcoma with epithelial neuroendocrine differentiation in the urinary bladder is unknown because of the extremely small number of reported cases. Our patient is alive with no evidence of disease eight months after diagnosis.

This represents the first reported case of a carcinosarcoma of the urinary bladder with a large cell neuroendocrine epithelial component. This rare tumour should be included in the differential diagnosis of biphasic neoplasms of the urinary bladder.

\section{Authors' affiliations}

Yu Li, S C Mathur, Department of Pathology, V A Medical Center, Kansas City, MO64128, USA

J E Outman, Department of Pathology, V A Medical Center

Correspondence to: Dr S C Mathur, Department of Pathology and Laboratory Medicine, V A Medical Center, 4801 Linwood Boulevard, Kansas City, MO 64128, USA; sharad.mathur@med.va.gov

Accepted for publication 21 October 2003

\section{REFERENCES}

1 Baschinsky DY, Chen JH, Vadmal MS, et al. Carcinosarcoma of the urinary bladder-an aggressive tumor with diverse histogenesis. Arch Pathol Lab Med 2000;24:1172-8.

2 Young RH. Carcinosarcoma of urinary bladder. Cancer 1987;59:1333-9.

3 Gronau S, Menz CK, Melzner I, et al. Immunohistomorphologic and molecular cytogenetic analysis of a carcinosarcoma of the urinary bladder. Virchows Arch 2002;440:436-40.

4 Halachmi S, DeMarzo AM, Chow N-H, et al. Genetic alterations in urinary bladder carcinosarcoma: evidence of a common clonal origin. Eur Urol 2000;37:350-7.

5 Young RH, Elbe JN. Unusual forms of carcinoma of the urinary bladder. Hum Pathol 1991;22:948-65.

6 Mazzucchelli L, Kraft R, Gerber H, et al. Carcinosarcoma of the urinary bladder: a distinct variant characterized by small cell undifferentiated carcinoma with neuroendocrine features. Virchows Arch A Pathol Anat Histopathol 1992;421:477-83.

7 Mills SE, Wolfe JT, Weiss MA, et al. Small cell undifferentiated carcinoma of the urinary bladder. A light-microscopic, immunocytochemical, and ultrastructural study of 12 cases. Am J Surg Pathol 1987;1 1:606-17.

8 Evans AJ, Al-Maghrabi J, Tsihlias J, et al. Primary large cell neuroendocrine carcinoma of the urinary bladder. Arch Pathol Lab Med 2002;126:1229-32.

9 Travis WD, Linnoila RI, Tsokos MG, et al. Neuroendocrine tumors of the lung with proposed criteria for large-cell neuroendocrine carcinoma: an ultrastructural, immunohistochemical, and flow cytometric study of 35 cases. Am J Surg Pathol 1991;15:529-53.

10 Murphy WM, Beckwith JB, Farrow GM. Atlas of tumor pathology: tumors of the kidney, bladder and related urinary structures. Washington, DC: Armed Forces Institute of Pathology, 1994. 